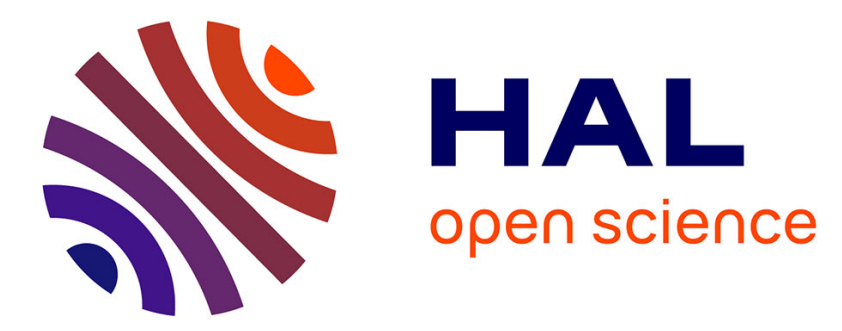

\title{
In depth static and low-frequency noise characterization of n-channel FinFETs on SOI substrates at cryogenic temperature
}

H. Achour, Bogdan Cretu, Jean-Marc Routoure, Régis Carin, Rachida Talmat, A. Benfdila, E. Simoen, C. Claeys

\section{To cite this version:}

H. Achour, Bogdan Cretu, Jean-Marc Routoure, Régis Carin, Rachida Talmat, et al.. In depth static and low-frequency noise characterization of n-channel FinFETs on SOI substrates at cryogenic temperature. Solid-State Electronics, 2014, 8 p. hal-00994380

\section{HAL Id: hal-00994380 \\ https://hal.science/hal-00994380}

Submitted on 21 Jul 2014

HAL is a multi-disciplinary open access archive for the deposit and dissemination of scientific research documents, whether they are published or not. The documents may come from teaching and research institutions in France or abroad, or from public or private research centers.
L'archive ouverte pluridisciplinaire HAL, est destinée au dépôt et à la diffusion de documents scientifiques de niveau recherche, publiés ou non, émanant des établissements d'enseignement et de recherche français ou étrangers, des laboratoires publics ou privés. 


\title{
In depth static and low-frequency noise characterization of n-channel FinFETs on SOI substrates at cryogenic temperature
}

\author{
H. Achour ${ }^{\mathrm{a}, \mathrm{d}}$, B. Cretu ${ }^{\mathrm{b}, \mathrm{c}, *}$, J.-M. Routoure ${ }^{\mathrm{a}, \mathrm{c}}$, R. Carin ${ }^{\mathrm{a}, \mathrm{c}}$, R. Talmat ${ }^{\mathrm{a}, \mathrm{d}}$, A. Benfdila ${ }^{\mathrm{d}}$, E. Simoen ${ }^{\mathrm{e}}$, C. Claeys $^{\mathrm{e}, \mathrm{f}}$ \\ ${ }^{a}$ University of Caen Basse-Normandie, UMR 6072 GREYC, F-14050 Caen, France \\ ${ }^{\mathrm{b}}$ ENSICAEN, UMR 6072 GREYC, F-14050 Caen, France \\ ${ }^{\mathrm{C}}$ CNRS, UMR 6072 GREYC, F-14032 Caen, France \\ d GRMNT, Mouloud Mammeri University of Tizi-Ouzou, Algeria \\ ${ }^{\mathrm{e}}$ Imec, Kapeldreef 75, B-3001 Leuven, Belgium \\ ${ }^{\mathrm{f}}$ E.E. Dept. KU Leuven, Kasteelpark Arenberg 10, B-3001 Leuven, Belgium
}

\author{
Keywords: \\ SOI FinFET \\ Strain \\ Very low temperature \\ Short channel effects \\ Analog parameters \\ Low frequency noise
}

\begin{abstract}
A B S T R A C T
The impact of cryogenic temperature operation $(10 \mathrm{~K})$ on the short channel effects and low frequency noise was analysed on strained and unstrained n-channel FinFET transistors fabricated on silicon on insulator (SOI) substrates in order to evaluate the devices static performances and to study the low frequency noise mechanisms. The main electrical parameters are investigated and it is evidenced that even at very low temperatures, the strain-engineering techniques boost the devices performances in terms of mobility, threshold voltage, access resistances and drain saturation currents. The DIBL effect, Early voltage and the intrinsic gain are ameliorated only for the short channel devices. A drawback, however, is that slightly improved turn-on capabilities may be noted for standard channel devices compared to strained ones. Low frequency noise measurements show that the carrier number fluctuations dominate the flicker noise in weak inversion even at $10 \mathrm{~K}$ operation. Access resistance noise contributions were evidenced in strong inversion.
\end{abstract}

\section{Introduction}

The development of low and very low temperature microelectronics is of considerable interest for the space industry, which is an important customer of cryogenic electronics since many satellites and space probes consist of a cryogenic enclosure which ensures to maintain the embedded devices at a constant very low temperature (e.g. $77 \mathrm{~K}$ and $4.2 \mathrm{~K}$ ). An advantage of the use of low temperature operation for specific applications is that the devices/circuit performances increase compared to room temperature operation. The temperature reduction leads to a higher speed due to improved transport properties and amelioration of turn-on capabilities. Other favourable aspects of the cryogenic operation are the disappearance of the thermally activated parasitic effects, the decrease of the leakage currents, and the reduction of the interconnection resistances, of the power consumption and of the thermal noise, respectively. Thus, one may note that the parasitic effects which are accompanying the continuous miniaturization

* Corresponding author at. ENSICAEN, UMR 6072 GREYC, F-14050 Caen, France. Tel.: +33 (0) 2314527 17; fax: +33 (0) 231452698 .

E-mail address: bogdan.cretu@ensicaen.fr (B. Cretu). of the devices can be controlled by reducing the operating temperature. However, some disadvantages, such as impurity freeze-out, access resistance effects, anomalies in the transient response etc., may appear at low temperature operation and may limit the functionality of the circuits. Other difficulties are associated with the changes in the fundamental mechanisms related with electronic transport and fluctuations in the devices [1-7].

The multi-gate FinfET has been considered as a promising structure for the future technology nodes, in particular since the 3D geometry may offer an improved control of the short-channel effects through a better electrostatic control of the gate over the conduction channel, giving the possibility to achieve a higher $I_{O N} / I_{O F F}$ ratio, and providing an enhanced mobility due to the undoped channel [8-11]. However, according to the International Technology Roadmap for Semiconductors (ITRS), the FinFETs still need to reach a higher $I_{O N}$ to meet the technology requirements. For the enhancement of the device mobility without adding major process complexity, strain-engineering techniques may be used [12-15].

This work is focused on a detailed characterization of unstrained and strained n-channel FinFETs on SOI substrates at very low temperature $(10 \mathrm{~K})$ operation in terms of short channel 
effects, analog operation parameters and low frequency noise performances. It is important to observe whether or not strainengineering is still useful at this cryogenic temperature.

\section{Devices and experimental procedures}

The studied devices were processed at imec in a $32 \mathrm{~nm}$ SOI technology with standard and strain-engineered channels. The tested n-channel FinFETs have a fin width $\left(W_{\text {Fin }}\right)$ of $25 \mathrm{~nm}$, fin height $\left(H_{\text {Fin }}\right)$ of $65 \mathrm{~nm}, 5$ fins $\left(N_{\text {Fin }}\right)$ in parallel and a mask gate length $\left(L_{\mathrm{G}}\right)$ varying from $0.13 \mu \mathrm{m}$ to $1 \mu \mathrm{m}$. The gate oxide consists of a high-k dielectric (HfSiON) on top of a $1 \mathrm{~nm}$ interfacial $\mathrm{SiO}_{2}$ resulting in an equivalent oxide thickness (EOT) of $1.5 \mathrm{~nm}$. The metal gate consists of $10 \mathrm{~nm}$ TiN covered by $100 \mathrm{~nm}$ polysilicon. The tested devices are on standard SOI substrates and on biaxial globally strained sSOI substrates combined with uniaxial local strain by CESL (Contact Etch Stop Layers) and using SEG (Selective Epitaxial Growth) in the drain and source regions $($ sSOI + CESL + SEG).

The static and the low frequency noise measurements were performed directly at wafer-level using a Lakeshore TTP4 prober. DC measurements were made using an HP4156B semiconductor parameter analyser.

In linear regime operation, the devices were biased with an applied drain voltage $V_{D S}=20 \mathrm{mV}$ for an applied gate voltage $V_{G S}$ which varies from $-0.5 \mathrm{~V}$ up to $1.5 \mathrm{~V}$. In the saturation regime, $I_{D}\left(V_{D S}\right)$ measurements were performed for different fixed applied gate voltage $V_{G S}$ from $0.6 \mathrm{~V}$ up to $1 \mathrm{~V}$, for an applied drain voltage $V_{D S}$ which varies from 0 up to $1.2 \mathrm{~V}$.

In the linear regime measurements were performed for all available gate lengths at $10 \mathrm{~K}, 80 \mathrm{~K}$ and at room temperature. The saturation regime operation was investigated for all available gate lengths at room temperature, while at $10 \mathrm{~K}$ the measurements were focused only on three mask gate lengths of $0.13 \mu \mathrm{m}$, $0.25 \mu \mathrm{m}$ and $0.7 \mu \mathrm{m}$.

The noise measurement set-up allows to bias the devices by choosing the $V_{G S}$ and $V_{D S}$ voltages, and also to measure the total dynamic resistance between drain and source $r_{T}$ and the transconductance $g_{m}$ by applying a small signal at the source and gate nodes, respectively. Drain current fluctuations are amplified and the noise spectral density is calculated using a FFT spectral analyser. Noise is reported at the input of the device by dividing by the square of the measured voltage gain between the gate and the output and this for different applied gate voltages. The low frequency noise measurements were focused only on two mask gate lengths $(0.2 \mu \mathrm{m}$ and $0.1 \mu \mathrm{m})$.

\section{Results and discussion}

\subsection{Static measurement}

Typical output transfer characteristics $I_{D}\left(V_{G S}\right), g_{m}\left(V_{G S}\right)$ and $I_{D}\left(V_{D S}\right)$ for standard and strained n-channel FinFETs at $10 \mathrm{~K}$ are shown in Fig. 1(a) and (b). Correct behaviours can be observed even for the shortest mask gate length and one can note that the benefit of the use of a strain-engineered channel seems to be preserved at this low temperature operation.

At temperatures lower than $40 \mathrm{~K}$, the effective mobility has a bell-shaped behaviour with the inversion charge [6,7]. In order to eliminate the effects of the mobility gate voltage dependence at $10 \mathrm{~K}$ operation, according to [16], an adapted function (noted $\left.Y_{10 K}\right)$ defined as $\left(I_{D}\right)^{2 / 3} /\left(g_{m}\right)^{1 / 3}$ should be constructed. In the inset of Fig. 1(a) is plotted the $Y_{10 K}$ function for the shortest mask gate length $\left(L_{G}=0.16 \mu \mathrm{m}\right)$ for both standard and strained channel. A linear dependence of this function with the applied gate voltage can be observed in strong inversion, as expected. Thereafter, by using the parameter extraction technique described in [16], the main electrical parameters of the studied devices can be estimated at $10 \mathrm{~K}$ operation. At room temperature and $80 \mathrm{~K}$ operation, the electrical parameters are extracted following the Y-function methodology proposed in [17]. It should be noted that, according to [16,17], the maximum of the effective mobility can be estimated at $10 \mathrm{~K}$, while at $80 \mathrm{~K}$ and $300 \mathrm{~K}$ it is the low field mobility. The obtained values are summarised in Table 1 . The impact of phonon scattering mechanisms decreases with the temperature reduction; this may explain the increase of the extracted mobility from $300 \mathrm{~K}$ down to $80 \mathrm{~K}$ for both studied structures. The impact of Coulomb and surface roughness scattering mechanisms increases at low temperature operation [18]; this could be associated to the reduction of the mobility at $10 \mathrm{~K}$. The benefit of a strain-engineered channel is evidenced by an enhancement of about $240 \%$ of the extracted low field mobility compared to standard ones at room temperature. This enhancement is reduced at $80 \mathrm{~K}$ operation (i.e. about $200 \%$ ). One should note that this enhancement of the mobility is drastically reduced at $10 \mathrm{~K}$ operation (i.e. about $133 \%$ ); this trend could be associated with a more pronounced impact of the scattering mechanisms (Coulomb and surface roughness) in strained structures at low temperature operation.

The extracted threshold voltage $\left(V_{T H}\right)$ values for the $0.14 \mu \mathrm{m}$ and $1 \mu \mathrm{m}$ mask gate length for standard and strained n-FinFETs are shown in Fig. 2. The solid line gives the expected behaviour of the threshold voltage increase with temperature reduction, which is modelled with a rate of about $-0.57 \mathrm{mV} / \mathrm{K}$ [19]. This trend is not respected at cryogenic temperature operation; at $10 \mathrm{~K}$ operation the values of the threshold voltage are lower than those at $80 \mathrm{~K}$. This could be related to the temperature behaviour of the Fermi level and of the surface potential at very low temperatures which are caused primarily by the temperature dependence of the intrinsic carrier concentration [20]. Moreover, the shift of the threshold voltage $\left|V_{T H}(300 \mathrm{~K})-V_{T H}(10 \mathrm{~K})\right|$ for the SOI devices is about $110 \mathrm{mV}$ for $L_{G}=1 \mu \mathrm{m}$ and $87 \mathrm{mV}$ for $L_{G}=0.14 \mu \mathrm{m}$, while in strained device it is about $41 \mathrm{mV}$ for $L_{G}=1 \mu \mathrm{m}$ and $32 \mathrm{mV}$ for $L_{G}=0.14 \mu \mathrm{m}$, resulting in a lower threshold voltage for the strained structures at $10 \mathrm{~K}$.

An important electrical parameter, in particular for dynamic switching circuits, is the subthreshold swing $S$, defined as the inverse of the slope of the $\log \left(I_{D}\right)$ versus $V_{G S}$ characteristics in the subthreshold region. Compared to the room temperature value, a theoretical reduction of the substhrehold swing with a factor 3.75 and 30 should be obtained at $80 \mathrm{~K}$ and $10 \mathrm{~K}$ operation, respectively. Substantial enhancement of the turn-on capabilities could then be obtained only by lowering the temperature. The extracted values of the subthreshold swing for a mask gate length of $0.2 \mu \mathrm{m}$ are summarised in Table 1 . The obtained subthreshold swing values present a slight degradation to the ideal values at room temperature and $80 \mathrm{~K}$ (i.e. $60 \mathrm{mV} / \mathrm{dec}$ and $16 \mathrm{mV} / \mathrm{dec}$, respectively). The difference observed between the expected and experimental values of the $S$ parameter at $10 \mathrm{~K}$ (ideal value of $2 \mathrm{mV} / \mathrm{dec}$, while experimental value are about $17 \mathrm{mV} / \mathrm{dec}$ and $14 \mathrm{mV} / \mathrm{dec}$ for strained and unstrained devices, respectively) may be related to an increase of the interface states at the band edges at very low temperatures [4]. Slightly improved turn-on capabilities may be noted for standard channel devices compared to strained ones at all investigated temperatures.

The access resistance $R_{\text {access }}$ leads to a decrease of the drain current of the devices compared to the drain current which can be obtained with an ideal device under the same bias conditions and becomes a major concern for scaled down devices. The increase of the parasitic access resistance impact on the output transfer performances is one of the drawbacks related with the use of narrow n-channel FinFETs. The access resistance and the 

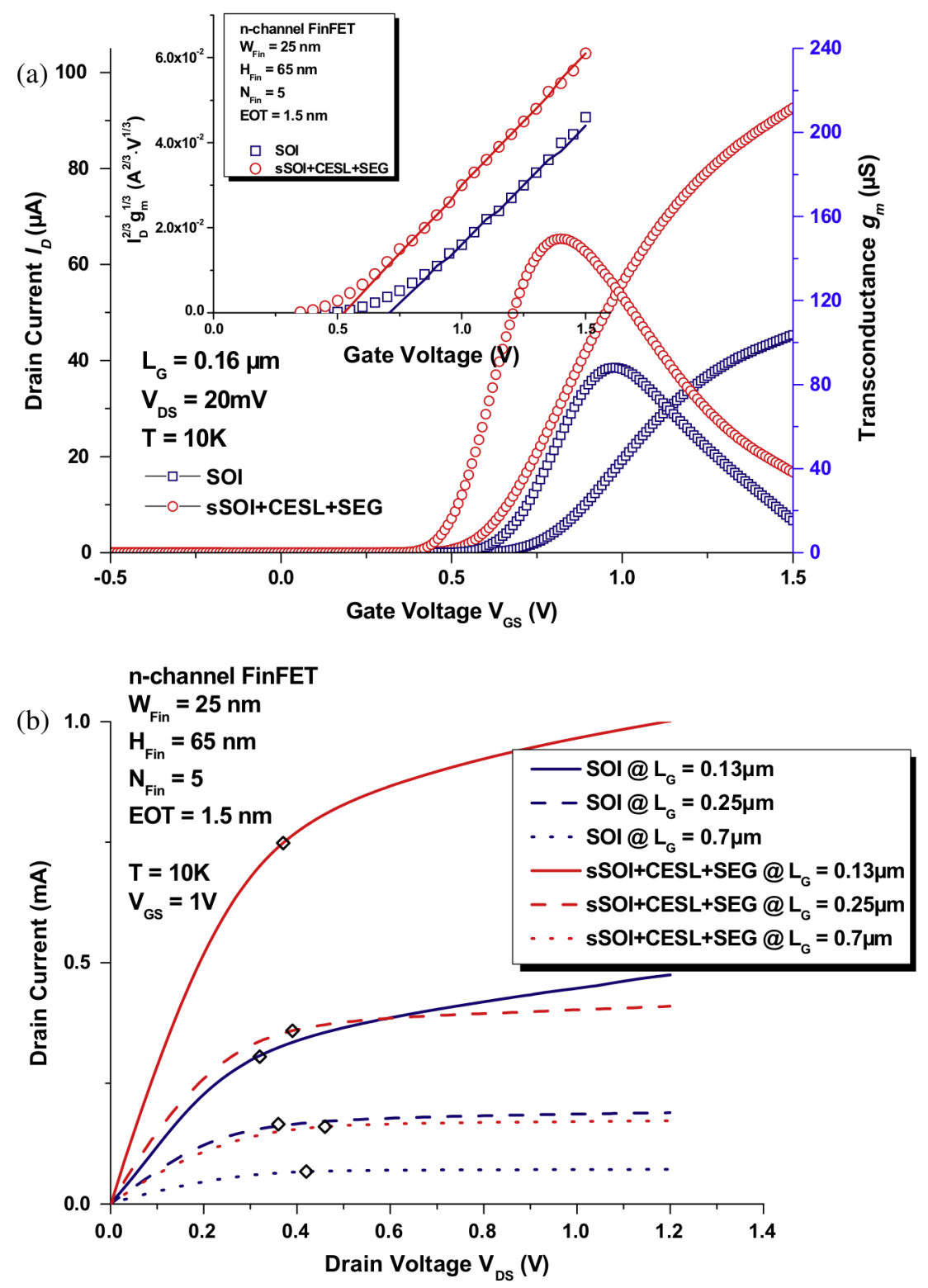

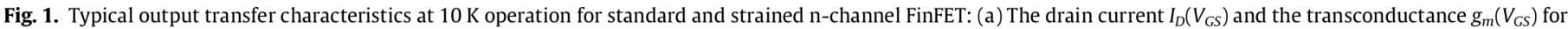

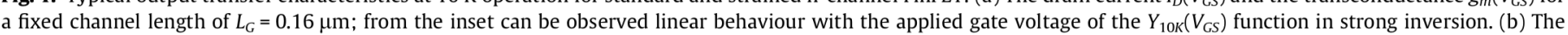
drain current $I_{D}\left(V_{D S}\right)$ for $L_{G}=0.13 \mu \mathrm{m}, L_{G}=0.25 \mu \mathrm{m}$ and $L_{G}=0.7 \mu \mathrm{m}$. The rhombus points represent the extracted $I_{D s a t}$ and $V_{D s a t}$ levels.

Table 1

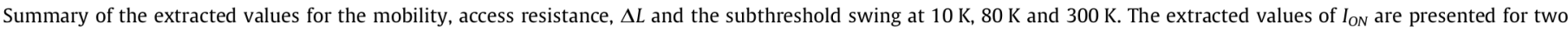
channel gate length at $10 \mathrm{~K}$ and $300 \mathrm{~K}$.

\begin{tabular}{|c|c|c|c|c|c|c|c|}
\hline & & \multicolumn{3}{|l|}{ SOI } & \multicolumn{3}{|c|}{$\mathrm{SSOI}+\mathrm{CESL}+\mathrm{SEG}$} \\
\hline & & $10 \mathrm{~K}$ & $80 \mathrm{~K}$ & $300 \mathrm{~K}$ & $10 \mathrm{~K}$ & $80 \mathrm{~K}$ & $300 \mathrm{~K}$ \\
\hline$\mu\left(\mathrm{cm}^{2} / \mathrm{Vs}\right)$ & & 490 & 593 & 220 & 653 & 1187 & 530 \\
\hline$R_{\text {access }}(\Omega)$ & & 178 & 181 & 210 & 84 & 82 & 123 \\
\hline$\Delta L(\mathrm{~nm})$ & & 18 & 37 & 54 & 22 & 26 & 49 \\
\hline$S(\mathrm{mV} / \mathrm{dec}) @ L_{G}=0.2 \mu \mathrm{m}$ & & 14.3 & 17.2 & 62.3 & 16.7 & 18.1 & 64.2 \\
\hline \multirow[t]{2}{*}{$I_{O N}(\mu \mathrm{A})$} & $0.7 \mu \mathrm{m}$ & 71 & & 61 & 170 & & 142 \\
\hline & $0.13 \mu \mathrm{m}$ & 447 & & 433 & 965 & & 894 \\
\hline
\end{tabular}

difference between the mask and the effective gate length ( $\Delta L=L_{G}-L_{e f f}$ ) of the studied devices are extracted following the total resistance technique described in [21]. The benefit of the integration of SiGe in the source and drain regions, realized by selective epitaxial growth is highlighted by the significant reduction of the access resistance in the devices that received SEG compared to standard ones with about $50-60 \%$ for all investigated temperatures (see Table 1 ). The decrease of the access resistance which can be observed from $300 \mathrm{~K}$ down to $80 \mathrm{~K}$ is more pronounced for strained devices (about 33\%) compared to standard 


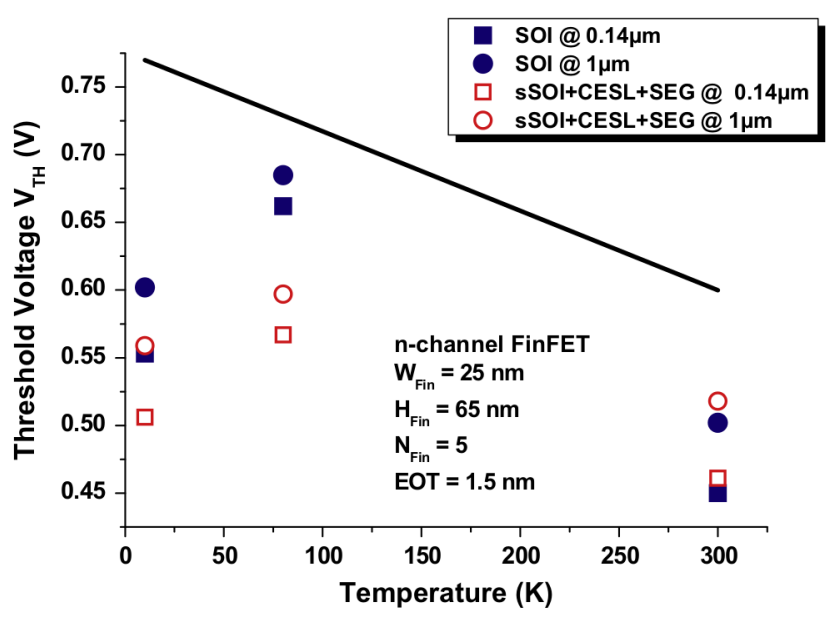

Fig. 2. Threshold voltage $V_{T H}$ versus the temperature for standard and strained devices for two different gate lengths $L_{G}=0.14 \mu \mathrm{m}$ and $L_{G}=1 \mu \mathrm{m}$. The solid line represents the expected increase of the threshold voltage with temperature reduction (rate of $-0.57 \mathrm{mV} / \mathrm{K}$ ).

ones (about 14\%). This trend seems not to be followed at very low temperatures: the extracted access resistance values at $10 \mathrm{~K}$ are near to those extracted at $80 \mathrm{~K}$. Increase of the access resistance at deep cryogenic operation was already reported and this behaviour is related to the impurity freeze-out of the lightly doped source and drain regions [5].

The effective channel length is a key MOSFET parameter since it is directly related to the current level supplied by the transistor. It is the only one (among the mask, physical (or poly), metallurgic and effective gate length) that can be directly extracted from electrical measurements. The estimated values of $\Delta L$ are summarised in Table 1. For a given temperature, the obtained values are quite similar for standard and strained devices. This suggests that the charge charring effect is not significantly affected by the use of strain. As expected, amelioration is observed by lowering the temperature operation: for the shortest channels available (i.e. $0.13 \mu \mathrm{m}$ ), the gate voltage controls effectively about $60 \%$ of the channel mask length at $300 \mathrm{~K}$ against about $85 \%$ at $10 \mathrm{~K}$.

The estimated values of the $I_{O N}$ current, defined as $I_{O N}=I_{D S} @ V_{D S}=V_{G S}=1 \mathrm{~V}$, are presented in Table 1 for a long and a short channel device at room temperature and $10 \mathrm{~K}$ operation. The enhancement of the $I_{O N}$ current with temperature reduction from $300 \mathrm{~K}$ down to $10 \mathrm{~K}$ is more pronounced for long-channel transistors (about 11-19\%) compared to short-channel transistors (about 4-8\%) for both structures. An $I_{O N}$ increase in strained devices compared to standard ones with a factor of about 2.4 and 2.1 can be observed even at $10 \mathrm{~K}$ operation for a long-channel and a short-channel, respectively.

The drift velocity $v_{D}$ increases with the drain voltage and reaches a maximum at high electric field. For an applied drain voltage higher than the saturation voltage, this maximum is weakly dependent on the applied gate bias. In order to eliminate the influence of the access resistance the following expression can be used for the drift velocity estimation: $v_{D}=g_{m} /\left[W C_{o x}\left(1-g_{m} R_{a c c e s s} / 2\right)\right]$ [21]. As expected from theory, $v_{D}$ is found to vary as the inverse of the channel length even for the short devices due to prevailing mobility limited transport (see Fig. 3a). The use of strain leads to enhanced values of the saturation velocity. Therefore, reducing the temperature increases the drift velocity for both devices due to the reduced phonon scattering contribution. It may be noted that at $10 \mathrm{~K}$, the estimated drift velocity for the shortest strained channel devices (about $10.9 \cdot 10^{6} \mathrm{~cm} / \mathrm{s}$ for $L_{G}=0.13 \mu \mathrm{m}$ ) is very close to the value of the non-stationary regime at this temperature (i.e. $13.2 \cdot 10^{6} \mathrm{~cm} / \mathrm{s}[22]$ ).
In the subthreshold operation, the drain induced barrier lowering effect (DIBL) leads to enhanced source injection resulting in an increased leakage current. The DIBL effect is generally studied by the threshold voltage reduction by increasing the drain voltage $\left(V_{T H}\left(V_{D S}\right)=V_{T H}-\lambda \cdot V_{D S}\right.$, where $\lambda$ is the DIBL parameter). As this short-channel effect results in a lowering of the source/ substrate barrier by applying a high drain voltage, we can estimate the DIBL effect by using the following parameter: $\mathrm{DIBL}=\left[\partial\left(\log I_{D S}\right) / V_{D S}\right]_{V_{G S=\text { const }}}$. The DIBL and $\lambda$ parameters are linked by the subthreshold slope. In Fig. 3(b) the extracted DIBL parameter at room temperature and at $10 \mathrm{~K}$ is plotted. For the shortest channel gate length, an enhancement of the DIBL effect is highlighted by the deviation to the expected variation with the inverse of the channel length (solid lines in Fig. 3b). This trend was already reported for advanced $50 \mathrm{~nm}$ nMOSFETs with $1.2 \mathrm{~nm}$ $\mathrm{SiO}_{2}$ dielectric [23]. However, this increase is more pronounced for the standard devices than for the strain-engineered ones. On the contrary, for long gate lengths the strained devices suffer more from the DIBL effect. The DIBL parameter $\lambda$, which can be calculated taking into account the extracted values for the subthreshold swing and the DIBL, is represented in the inset of Fig. $3(\mathrm{~b})$. The amelioration of the DIBL effect, which can be observed at very low temperature operation (i.e. $10 \mathrm{~K}$ ), seems to be more significant for short channels (reduction of $\lambda$ with a factor of about 6 for $L_{G}=0.13 \mu \mathrm{m}$ against a factor of about 2 for $L_{G}=0.7 \mu \mathrm{m}$ for both structures). Because this parasitic effect is essentially electrostatic, one expects that the DIBL parameter should be nearly temperature insensitive [24]. However, reduction of the DIBL at lower temperatures was already reported for small geometry devices $[25,26]$.

The obtained values of the absolute Early voltage $V_{E A}$ parameter, defined as $V_{E A} \cong I_{D S} / g_{D S}$, ( $g_{D S}$ being the drain conductance measured in saturation at $V_{D S}=1.2 \mathrm{~V}$ and $V_{G S}=1 \mathrm{~V}$ ) for our devices are shown in the Fig. 3(c). At cryogenic temperature operation, an increase with a factor of about 2 of $V_{E A}$ is observed in strain-engineered devices compared to standard ones for $L_{G}=0.13 \mu \mathrm{m}$. This benefit is lost for $L_{G}=0.25 \mu \mathrm{m}$ and $L_{G}=0.7 \mu \mathrm{m}$, for which is evidenced a degradation with a factor about 1.3 and 1.6, respectively. At room temperature operation, the same behaviour is observed; the benefit of using a strained channel is kept for gate length lower than $L_{G}=0.5 \mu \mathrm{m}$. Except for the $L_{G}=0.13 \mu \mathrm{m}$ strained device, the operation at $10 \mathrm{~K}$ seems to lead to a degradation of the Early voltage for both structures. Linear dependence of the $V_{E A}$ with the mask gate length can be observed from Fig. 3(c) for standard devices, resulting in a ratio of about $60 \mathrm{~V} / \mu \mathrm{m}$ at room temperature, which exceeds the normally reported values for conventional FD SOI [27]. The same ratio (at $300 \mathrm{~K}$ ) could be observed also for strained devices by taking into account only the shortest channel length $(\leqslant 0.35 \mu \mathrm{m})$.

Another common analog figure of merit is the transistor intrinsic gain $A_{V}$, defined as $A_{V}=V_{E A} \cdot g_{m} / I_{D S}$. Using the obtained values of $V_{E A}$ the intrinsic gain determined at $10 \mathrm{~K}$ and at room temperature are presented in the inset of Fig. 3(c) as a function of the gate length $L_{G}$. The obtained values are of the same order of magnitude than already reported for a similar FinFET technology [28]. For long channel devices $\left(L_{G}=0.7 \mu \mathrm{m}\right)$ the intrinsic gain is about $5.7 \mathrm{~dB}$ higher for unstrained devices compared to strained ones at $10 \mathrm{~K}$, which is about $1 \mathrm{~dB}$ higher that at room temperature operation. With the mask gate length $L_{G}$ downscaling, this difference reduces and for $L_{G}=0.13 \mu \mathrm{m}$ an improvement of about $2.1 \mathrm{~dB}$ (which is $0.4 \mathrm{~dB}$ higher than at $300 \mathrm{~K}$ ) can be observed for strained devices against the standard ones.

\subsection{Low frequency noise at $10 \mathrm{~K}$}

Typical frequency normalized gate voltage noise spectral density is plotted in Fig. 4(a). One can notice that the noise spectral 

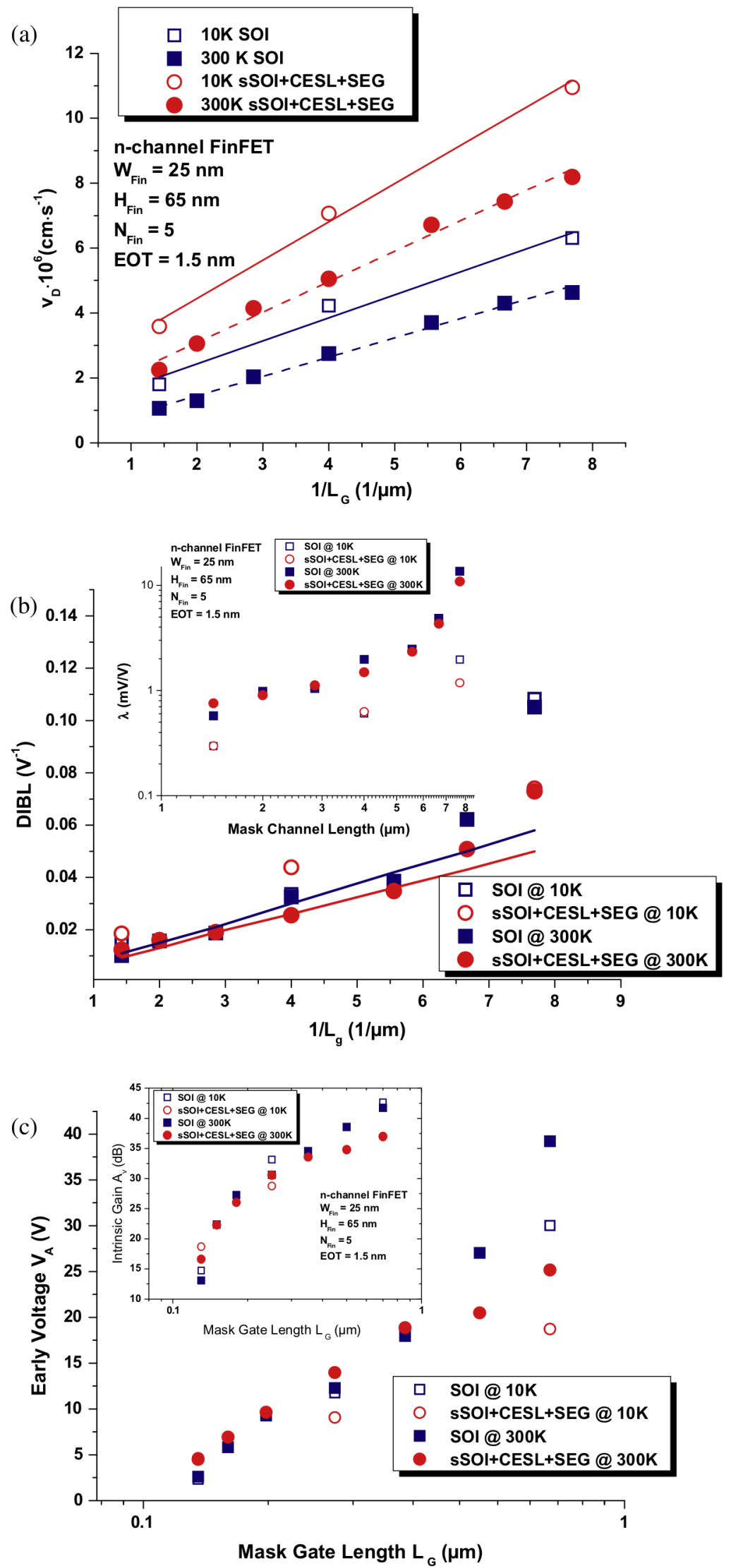

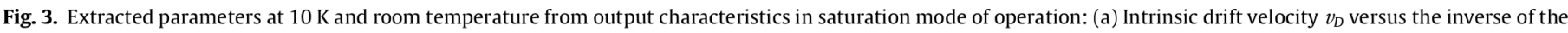

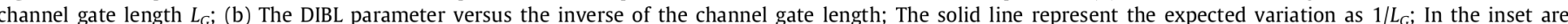

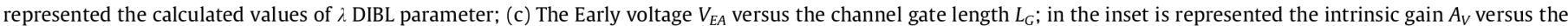
channel gate length. 

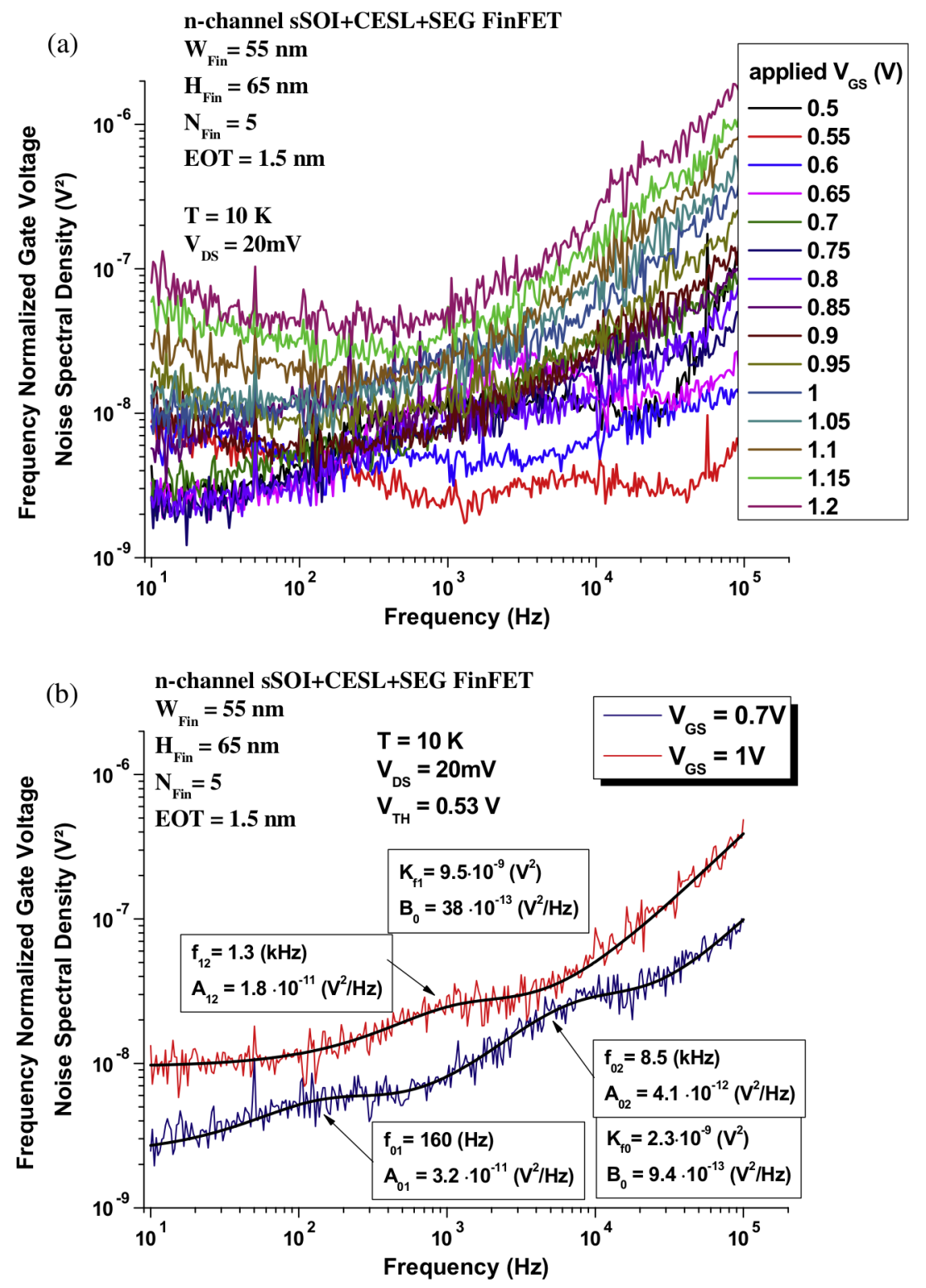

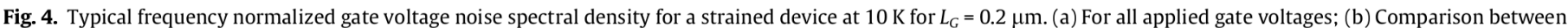

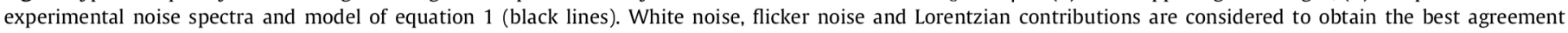
between the experimental noise spectra and model.

density contains both $1 / f$ and Lorentzian contributions. Generally, the frequency dependence of the different contributions on the total noise spectral density at the input of a MOS transistor can be expressed as:

$S_{V_{G}}(f)=B+\frac{K_{f}}{f}+\sum_{i=1}^{N} \frac{A_{i}}{1+\left(\frac{f}{f_{0 i}}\right)^{2}}$.

where $B$ is the white noise level, $K_{f} \mid f$ is the flicker noise and the third term of the equation presents a sum of Lorentzian components, with $A_{i}$ the plateau value and $f_{0 i}$ the characteristic frequency. As shown in Fig. 4(b), Eq. (1) permits to perfectly model the experimental noise spectra, allowing to identify the different noise parameters.

The extracted $1 / f$ noise level $\left(K_{f}\right)$ variations with the applied gate voltage overdrive $\left(V_{G T}=V_{G S}-V_{T H}\right)$ are illustrated in Fig. 5 for the two investigated mask gate lengths of the standard and strained devices at $10 \mathrm{~K}$ operation. At this cryogenic temperature, the extracted $1 / f$ noise levels are found to be independent of the applied gate overdrive in weak inversion for both structures. This suggests that carrier number fluctuations due to electron trapping in the oxide dominate at this cryogenic temperature for both structures. Therefore, the carrier number fluctuation model $[29,30]$ can be used to explain the origin of the noise in weak inversion and to extract an average oxide trap density $N_{i t}\left(/ \mathrm{eV} / \mathrm{cm}^{3}\right)$ (see Table 2). Assuming uncorrelated noise sources in the channel and source/ drain regions, the total $1 / f$ voltage noise spectral density in the linear region of operation can be described by the following equation $[21,31,32]$ :

$\frac{K_{f}}{f}=\frac{\left(r_{T}-r_{\text {access }}\right)^{2}}{r_{T}^{2}} S_{V_{F B}}+\frac{K_{r}}{f} \frac{r_{a c c e s s}^{2}}{2 r_{T}^{2}} \frac{I_{D}^{2}}{g_{m}^{2}}$

where $K_{r}$ is the access resistance noise level. From Fig. 5 one can observe a good correlation between the experimental points and the model of Eq. (2). This suggests that the increase of the noise 


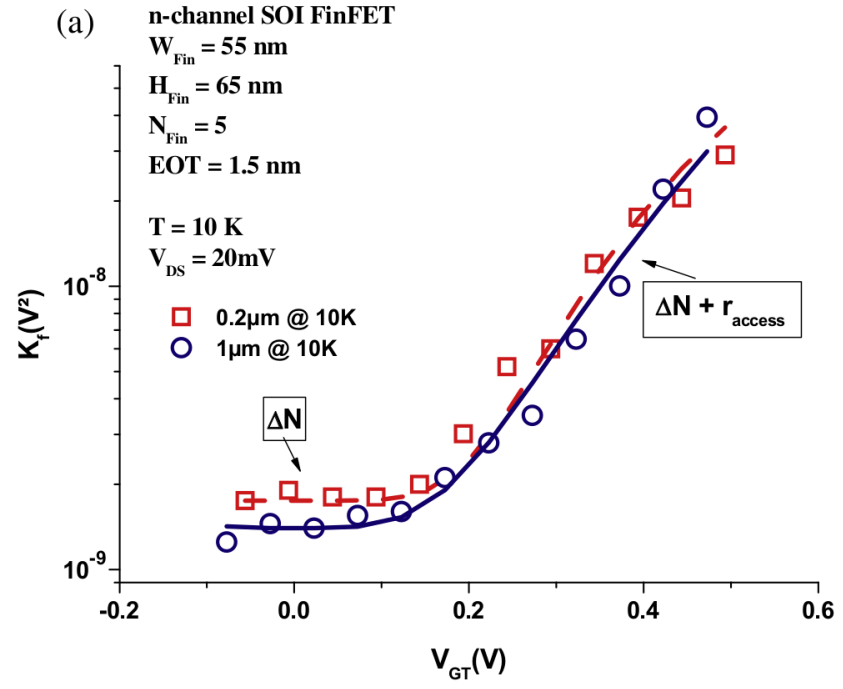

(b) n-channel sSOI+CESL+SEG FinFET

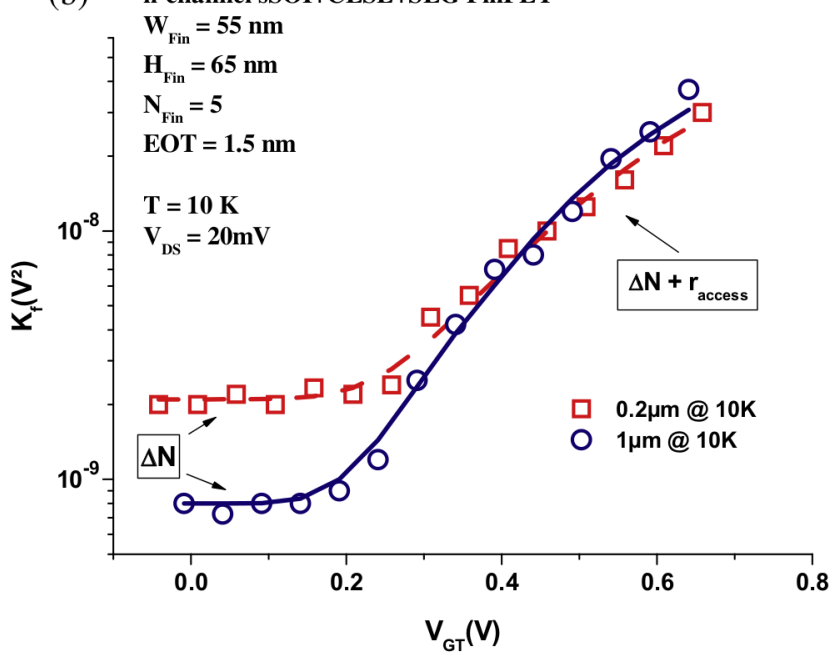

Fig. 5. The extracted $1 / f$ noise level $K_{f}$ at $10 \mathrm{~K}$ versus the applied gate overdrive $V_{G T}=V_{G S}-V_{T H}$ for $L_{G}=0.2 \mu \mathrm{m}$ and $L_{G}=1 \mu \mathrm{m}$. (a) Standard devices. (b) Strained devices. Good correlation between the experimental data and model of Eq. (2) is observed.

in strong inversion can be explained by the parasitic access resistance contributions.

In Table 2 are summarised the main extracted noise parameters at $10 \mathrm{~K}$ and $300 \mathrm{~K}$. It was already reported that reducing the temperature can lead to enhanced noise levels in n-MOSFETs [33]. An increase of the flat-band noise level is observed in our devices with temperature reduction from $300 \mathrm{~K}$ down to $10 \mathrm{~K}$. This increase is more pronounced for strained devices (a factor of about
4.6) compared to unstrained ones (a factor of 1.8) for short-channel devices (e.g. $\left.L_{G}=0.2 \mu \mathrm{m}\right)$, while for the long channel devices $\left(L_{G}=1 \mu \mathrm{m}\right)$ this increase seems to be not affected by the use of the strain (a factor of about 2.7 for unstrained devices against a factor of about 2.5 for the strained ones).

Except for the $L_{G}=0.2 \mu \mathrm{m}$ at $10 \mathrm{~K}$, a lowering of the $1 / \mathrm{f}$ noise level is observed in the studied devices with a sSOI substrate compared to standard ones. This reduction is in agreement with other reported experimental data [34-36]. The relatively small values of the slow insulator trap density are a good indication of the quality of the oxidation process despite the use of high- $k$ dielectrics in such advanced devices. By lowering the temperature down to $10 \mathrm{~K}$, an increase of the trap densities is observed. This behaviour was already reported [33] and can be explained by models which take into account the structure of the high-k dielectric stack considering two tunnelling barriers through the dielectric, corresponding to the interfacial layer and to the high- $k$ layer, respectively [37-39].

It should be noticed that the impact of the carrier number fluctuations correlated to mobility fluctuations is no more observed at $10 \mathrm{~K}$.

The access resistance noise levels are quite similar for both structures for $L_{G}=1 \mu \mathrm{m}$. On the contrary, a reduction of the $K_{r}$ level with a factor of about 3 is observed for strained devices compared to unstrained ones. These may corroborate with the fact that the access resistance impact is more important for scaled down devices and with the access resistance reduction due to the use of SEG in the strained devices.

\section{Conclusions}

A survey of the short channel effects in standard and strained nFinFETs at cryogenic temperatures has been given. The benefit of the use of strain engineering techniques is kept at very low temperatures: boosted carrier mobility, lower threshold voltage, reduced access resistance, higher $I_{O N}$ current and saturation drift velocity. The saturation velocity is near the non-stationary regime for the short-channel strained devices. The use of strain techniques can lead to opposite behaviours with the channel length reduction: amelioration of the DIBL effect, improvement of the Early voltage and of the intrinsic gain performances can be observed only for short-channel devices. Another drawback is the slight degradation of the turn-on capabilities for strain-engineered devices compared to standard ones for all investigated temperatures. Very low temperature operation improves the DIBL effect for both structures.

A lower $1 / f$ noise amplitude was found for devices on sSOI substrates compared to SOI ones. The carrier number fluctuations explain the $1 / f$ noise at cryogenic temperature in weak inversion. In strong inversion, the access resistance contribution prevails on the total $1 / f$ noise. No correlated mobility fluctuations contributions are observed at $10 \mathrm{~K}$. A lower access resistance noise level was found for short-channel devices which received SEG. The

Table 2

Summary of the extracted noise parameters for standard and strained devices at $10 \mathrm{~K}$ and $300 \mathrm{~K}$.

\begin{tabular}{|c|c|c|c|c|c|c|}
\hline & $T(\mathrm{~K})$ & $L_{G}(\mu \mathrm{m})$ & $S_{V F B} \cdot 10^{-9}\left(\mathrm{~V}^{2} / \mathrm{Hz}\right)$ & $K_{r} \cdot 10^{-5}$ & $\alpha_{s c} \cdot 10^{4}(\mathrm{Vs} / \mathrm{C})$ & $N_{i t} \cdot 10^{17}\left(/ \mathrm{eV} / \mathrm{cm}^{3}\right)$ \\
\hline \multirow[t]{4}{*}{ SOI } & 10 & 0.2 & 1.75 & 1.5 & & 610 \\
\hline & & 1 & 1.25 & 18 & & 2630 \\
\hline & 300 & 0.2 & 0.96 & 0 & 0.5 & 11 \\
\hline & & 1 & 0.45 & 6.5 & 0.14 & 29 \\
\hline \multirow[t]{4}{*}{$\mathrm{sSOI}+\mathrm{CESL}+\mathrm{SEG}$} & 10 & 0.2 & 2.1 & 0.55 & & 730 \\
\hline & & 1 & 0.72 & 19 & & 1490 \\
\hline & 300 & 0.2 & 0.45 & 0 & 0.35 & 5.1 \\
\hline & & 1 & 0.28 & 0 & 0.3 & 17 \\
\hline
\end{tabular}


relative small values of the oxide trap density highlight the quality of the oxidation process.

\section{References}

[1] Ghibaudo G, Balestra F, Emrani A. A survey of MOS devices physics for low temperature electronics. Microelectron Eng 1992;19:833-47.

[2] Balestra F, Ghibaudo G. Brief review of the MOS device physics for low temperature electronics. Solid State Electron 1994;17:1967-75.

[3] Simoen E, Dierickx B, Warmerdam L, Vermeiren J, Claeys C. Freeze-out effects on NMOS transistors characteristics at $4.2 \mathrm{~K}$. IEEE Trans Electron Dev 1989;36:1155.

[4] Hafez IM, Ghibaudo G, Balestra F. Assessment of interface state density in silicon metal oxide semiconductor transistors at room, liquid nitrogen, and liquid helium temperatures. J Appl Phys 1990;67:1950.

[5] Hafez IM, Ghibaudo G, Balestra F, Haond M. Impact of LDD structures on the operation of silicon MOSFETs at low temperature. Solid State Electron 1995;38:419-24.

[6] Ghibaudo G, Balestra F. Modelling of ohmic MOSFET operation at very low temperature. Solid State Electron 1988;31:105-8.

[7] Ghibaudo G, Balestra F. Low temperature characterisation of silicon CMOS devices. Microelectron Reliab 1997;37:1353-66.

[8] Hisamoto D, Lee WD, Jeon C, Kedzierski J, Takeuchi H, Asano K, et al. FinFET - a self-aligned double-gate MOSFET scalable to $20 \mathrm{~nm}$. IEEE Trans Electron Dev 2000;47:2320-5.

[9] Huang XJ, Lee WC, Kuo C, Hisamoto D, Chang L, Kedzierski J. Sub-50 nm Pchannel FinFET. IEEE Trans Electron Dev 2001:48:880-6.

[10] Colinge J. Multi-gate SOI, MOSFETs. Microelectron Eng 2007;84:2071-6.

[11] Skotnicki T. Materials and device structures for sub-32 nm CMOS nodes. Microelectron Eng 2007;84:1845-52.

[12] Parton E, Verheyen P. Strained silicon - the key to sub-45 nm CMOS. III-Vs review. Adv Semiconduct Mag 2006;19:28-32.

[13] Lai CM, Fang YK, Lin CT, Hsu CW, Yeh WK. The impacts of high tensile stress CESL and geometry design on device performance and reliability for $90 \mathrm{~nm}$ SOI nMOSFETs. Microelectron Reliab 2007;47:944-52.

[14] Collaert N, Rooyackers R, De Keersgieter A, Leys FE, Cayrefourq I, Ghyselen B, et al. Stress hybridization for multigate devices on supercritical strained-SO (SC-SSOI). IEEE Electron Dev Lett 2007;28:646-8.

[15] Claeys C, Simoen E, Put S, Giusi G, Crupi F. Impact strain engineering on gate stack quality and reliability. Solid State Electron 2008;52:1115-26.

[16] Ghibaudo G, Balestra F. A method for MOSFET parameter extraction at very low temperature. Solid State Electron 1989;32:221-3.

[17] Ghibaudo G. A new method for the extraction of MOSFET parameters. Microelectron Eng 1998;24:543.

[18] Jeon D, Burk D. MOSFET electron inversion layer mobilities - a physically based semi-empirical model for a wide temperature range. IEEE Trans Electron Dev 1989;36:1456-63.

[19] Talmat R, Put S, Collaert N, Mercha A, Claeys C, Guo W, Cretu B, Benfdila A, Routoure J-M, Carin R, Simoen E. High-temperature characterization of advanced strained nMuGFETs. In: Proceedings of EUROSOI'2010 conference 25-27 January Grenoble France; 2010. p. 75-6.

[20] Sze SM. Semiconductor devices - physics and technology. 2nd ed. John Wiley \& Sons Inc.; 2002.

[21] Ghibaudo G. Critical MOSFETs operation for low voltage/low power IC's: ideal characteristics parameter extraction electrical noise and RTS fluctuations. Microelectron Eng 1997:39:31-57.

[22] Jacoboni C, Canali C, Ottaviani G, Quaranta AA. A review of some charge transport properties of silicon. Solid State Electron 1977;20:77-89.

[23] Cretu B, Balestra F, Ghibaudo G, Guégan G. Low temperature operation of ultra-thin gate oxide sub-0.1 $\mu \mathrm{m}$ MOSFETs. J Phys IV France 2002;12:57.

[24] Yan ZX, Dee MJ. Substrate bias effects on drain-induced barrier lowering in short channel PMOS devices at 77 K. Cryogenics 1990;30:1160-5.

[25] Yan ZX, Dee MJ. Substrate bias effects on drain-induced barrier lowering in short channel PMOS devices at $77 \mathrm{~K}$. Cryogenics 1990;30:1160-5.

[26] Woo JCS, Plummer JD. Short-channel effects in MOSFET's at liquid nitrogen temperature. IEEE Trans Electron Dev 1986;33:1012-9.

[27] Lederer D, Kilchytska V, Rudenko T, Collaert N, Flandre D, Dixit A, et al. FinFET analogue characterization from DC to $110 \mathrm{GHz}$. Solid State Electron 2005;49:1488-96.

[28] Pavanello MA, Martino JA, Simoen E, Rooyackers R, Collaert N, Claeys C. Evaluation of triple-gate FinFETs with $\mathrm{SiO}_{2}-\mathrm{HfO}_{2}-\mathrm{TiN}$ gate stack under analog operation. Solid State Electron 2007;51:285-91.

[29] McWhorter. Semiconductor surface physics. Pensylvania: Pensylvania Press University; 1957.

[30] Ghibaudo G, Roux O, Nguyen-Duc C, Balestra F, Brini J. Low frequency noise in field-effect MOS transistors. Phys Stat Sol (a) 1991;124:571-81.

[31] Li X, Vandamme L. 1/f noise in series resistance of LDD MOSTs. Solid State Electron 1992;35:1477.

[32] Hooge FN. 1/f noise sources. IEEE Trans Electron Dev 1994;41:1926-35.

[33] Christensson S, Lundstrom I. Low-frequency noise in MOS transistors-II. Experiments. Solid State Electron 1968;11:813-20.

[34] Talmat R, Achour H, Cretu B, Routoure J-M, Benfdila A, Carin R, et al. Low frequency noise characterization in n-channel FinFETs. Solid State Electron 2012;70:20-6.

[35] Simoen E, Claeys C. Impact of strain-engineering on the low-frequency noise performance of advanced MOSFETs. In: ICSICT'2006; p. 120-3.

[36] Simoen E, Eneman G, Verheyen R, Loo R, De Meyer K, Claeys C. Processing aspects in the low-frequency noise of nMOSFETs on strained-silicon substrates. IEEE Trans Electron Dev 2006;53:1039.

[37] Morshed T, Devireddy SP, Rahman MS, Çelik-Butler Z, Tseng H, Zlotnicka A, Shanware A, Green K, Chambers JJ, Visokay MR, Quevedo-Lopez MA, Colombo L. A new model for $1 /$ f noise in high-k MOSFETs. In IEDM Tech, Dig.; 2007; p. 561-4.

[38] Morshed T, Devireddy SP, Çelik-Butler Z, Shanware A, Green K, Chambers JJ, et al. Physics-based $1 / \mathrm{f}$ noise model for MOSFETs with nitrided high-k gate dielectrics. Solid State Electron 2008:52:711-24.

[39] Hung K, Ping K, Chenming H, Cheng YC. A unified model for the flicker noise in metal-oxide-semiconductor field-effect transistor. IEEE Trans Electron Dev $1990 ; 37: 654-65$. 